\title{
Índice tornozelo-braço em pacientes hemodialíticos
}

\author{
Ankle-brachial index in hemodialysis patients
}

Mariane Torres Uchôa', Diego Nunes de Albuquerque Oliveira², Maria Eliete Pinheiro ${ }^{3}$,
Daniella Bezerra Duarte', Jairo Calado Cavalcante', Glauber Schettino Silva', Marcos Mota Gomes ${ }^{7}$

\section{Resumo}

Introdução: Apesar da elevada mortalidade dos pacientes em hemodiálise devido às doenças cardiovasculares, é incomum a realização de exames diagnósticos para doença arterial obstrutiva periférica e artérias pouco compressíveis.

Objetivos: Analisar a prevalência de doença arterial obstrutiva periférica e artérias pouco compressíveis em hemodialisados, comparando-os com o Grupo Controle. Métodos: Tratou-se de um estudo transversal, com 78 hemodialisados e 88 pacientes que não faziam hemodiálise com, pelo menos, dois fatores de risco para doença arterial obstrutiva periférica (Grupo Controle). Para aferição da pressão arterial sistólica, utilizou-se Doppler vascular portátil e esfigmomanômetro. Esta foi aferida somente nos membros que não possuíam fístula arteriovenosa. O índice tornozelo-braço foi calculado utilizando cada artéria do membro inferior. Foram considerados normais os valores de 0,9 a 1,3.

Resultados: Diagnosticou-se doença arterial obstrutiva periférica e artérias pouco compressíveis em 26,9 e 30,8\%, dos hemodialisados, e em 33 e 22,7\%, do Grupo Controle. Nos hemodialisados, verificou-se o índice tornozelo-braço alterado em 75\% dos sintomáticos ( $p=0,005)$, em 67,3\% dos homens e $31 \%$ das mulheres ( $p<0,005$ ), em 78,6\% dos idosos, 34,8\% dos adultos jovens ( $p<0,01)$ e em 76,9\% dos diabéticos ( $p<0,005$ versus não diabéticos). Esses pacientes apresentaram maior prevalência de doença arterial obstrutiva periférica grave do que o Grupo Controle $(p<0,01)$.

Conclusões: $\mathrm{O}$ índice tornozelo-braço anormal foi muito prevalente nos grupos estudados; entretanto, os hemodialisados apresentaram alterações mais graves quando comparados ao Grupo Controle. Diabetes melito, sexo masculino e idade avançada foram fatores de risco importantes para a alteração do índice tornozelo-braço nos hemodialisados. O índice tornozelo-braço foi um bom método de rastreio para alterações pesquisadas. Portanto, a utilização deste na rotina de manejo de pacientes em hemodiálise é sugerida.

Palavras-chave: índice tornozelo-braço; diálise; doenças vasculares periféricas.

\begin{abstract}
Introduction: Despite the high mortality in hemodialysis patients due to cardiovascular diseases, it is unusual performing diagnostic tests for peripheral arterial disease and poorly compressible arteries.

Objectives: To analyze the prevalence of peripheral arterial disease and poorly compressible arteries in hemodialysis patients, by comparing them to the Control Group.

Methods: It was a cross-sectional study with 78 hemodialysis patients and 88 non-hemodialysis patients with at least two risk factors for peripheral arterial disease (Control Group). To evaluate blood pressure, portable vascular Doppler and sphygmomanometer were used. The pressure was measured from the limb without arteriovenous fistula. The ankle-brachial index was calculated for each artery of the lower limb. It was considered normal ankle-brachial index from 0.9 to 1.3.

Results: We diagnosed peripheral arterial disease and poorly compressible arteries in 26.9 and $30.8 \%$ of hemodialysis patients and in 33 and $22.7 \%$ of the Control Group. In hemodialysis patients, we found abnormal ankle-brachial index in $75 \%$ of symptomatic patients ( $p=0.005$ ), in $67.3 \%$ of men and $31 \%$ of women $(p<0.005)$, in $78.6 \%$ of the elderly, $34.8 \%$ of young adults $(p<0.01)$, and $76.9 \%$ of diabetics ( $p<0.005$ versus nondiabetic). These patients had a higher prevalence of severe peripheral arterial disease than the Control Group.

Conclusions: Abnormal ankle-brachial index was very prevalent in the groups studied; however, hemodialysis patients had more severe changes, when compared to the Control Group. Diabetes mellitus, male gender, and advanced age were important risk factors for abnormal ankle-brachial index in hemodialysis patients. The ankle-brachial index was a good method of screening for the studied changes; therefore, we suggest the routine use in the management of hemodialysis patients.
\end{abstract}

Keywords: ankle-brachial index; dialysis; peripheral vascular diseases.






\section{Introdução}

Apesar da elevada mortalidade de pacientes em hemodiálise devido às doenças cardiovasculares, é incomum a realização de exames diagnósticos para artérias pouco compressíveis e doença arterial obstrutiva periférica (DAOP), as quais apresentam elevada prevalência em pacientes com doença renal crônica ${ }^{1-9}$.

O índice de mortalidade por doenças cardiovasculares em hemodialisados é de 5 a 30 vezes maior do que na população geral ${ }^{10}$, sendo a DAOP um fator preditor independente da ocorrência de insuficiência cardíaca ${ }^{11}$.

Algumas associações científicas discordam que a doença renal crônica implique em riscos para a incidência de $\mathrm{DAOP}^{12}$. Contudo, a maioria dos autores da literatura confirma e enfatiza a importância de tal relaçãa ${ }^{2,13}$.

Apesar da polêmica sobre a consideração da doença renal crônica como condição de risco para a DAOP, são quase inexistentes estudos que possibilitem a resolução deste impasse e que avaliem conjuntamente tais enfermidades em hemodialisados. As recomendações para triagem de DAOP em doentes renais crônicos na diretriz Kidney Disease Outcomes Quality Initiative (K/DOQI), por exemplo, são baseadas em estudos com pacientes pré-dialíticos e nos pareceres de especialistas ${ }^{1,13}$.

Tanto a DAOP quanto as artérias pouco compressíveis estão relacionadas com o aumento de mortalidade (cardiovascular ou por outras causas), em portadores de doença renal crônica ${ }^{14,15}$. Em hemodialisados, este aumento está presente mesmo em portadores assintomáticos de $\mathrm{DAOP}^{1}$.

A pesquisa de sintomas e a palpação de pulsos das artérias dos membros inferiores são ineficazes para o rastreio das doenças citadas.

O índice tornozelo-braço (ITB) é um método de baixo custo e não invasivo ${ }^{8}$, que apresenta $95 \%$ de sensibilidade e $99 \%$ de especificidade para DAOP, quando comparado à angiografia (padrão-ouro) ${ }^{2}$. Valores anormais do ITB estão relacionados a uma maior morbimortalidade ${ }^{7,16}$, sendo considerado forte preditor prognóstico.

A mensuração do ITB em hemodialisados não é realizada rotineiramente. Desta forma, as prevalências da DAOP e de artérias pouco compressíveis não estão satisfatoriamente documentadas.

O objetivo do presente estudo foi analisar a prevalência da DAOP e de artérias pouco compressíveis em um grupo de hemodialisados, comparando os dados encontrados com os de um Grupo Controle de alto risco para as duas patologias avaliadas. Além disso, foram relacionados os valores de ITB com fatores de risco e sinais ou sintomas apresentados pelos pacientes com doença renal crônica.

\section{Métodos}

Tratou-se de um estudo transversal, realizado com 78 hemodialisados, provenientes de um serviço público e um privado de Nefrologia, e 88 pacientes que não faziam hemodiálise e eram oriundos de um serviço privado de Cardiologia (Grupo Controle).

Foram incluídos, no grupo de hemodialisados: pacientes com mais de 18 anos em terapia renal substitutiva por hemodiálise, com fístula arteriovenosa funcionante em apenas um dos membros superiores, que não estavam fazendo hemodiálise por doença renal aguda e não apresentavam amputações de ambos os membros inferiores ou do membro superior contralateral à fístula.

No Grupo Controle, foram incluídos pacientes maiores de 18 anos, com pelo menos dois fatores de risco para DAOP, que não apresentavam amputações dos dois membros superiores ou inferiores e que não apresentavam fístulas arteriovenosas ou doença renal crônica.

Foram convidados a participar da pesquisa os pacientes das três instituições de saúde que respeitavam os critérios de inclusão.

Foram excluídos da pesquisa dois pacientes por se recusarem a serem examinados; um que se queixou de dor intensa ao ser mensurada as pressões arteriais de seus membros inferiores e outro, pela ausência da pressão do membro superior nos registros da pesquisa.

O projeto foi aprovado pelo Comitê de Ética em Pesquisa da Universidade à qual o estudo está vinculado (processo 009707/2010-11). Os pacientes assinaram o termo de consentimento livre e esclarecido antes da coleta de dados.

$\mathrm{Na}$ entrevista com os indivíduos do Grupo Controle, foram colhidos dados demográficos e realizaram-se indagações a respeito dos medicamentos utilizados e da presença de comorbidades.

Com o grupo de hemodialisados, foram coletadas as informações descritas, além de sinais e/ou sintomas indicativos de DAOP (dor em repouso, claudicação intermitente, sinais de hipoperfusão, história de revascularização de membro inferior e necrose ou amputação - de dedos, pés ou um dos membros inferiores).

Foram considerados hipertensos pacientes com diagnóstico de hipertensão arterial sistêmica, registrado em prontuário, aqueles com medida de pressão arterial maior que $140 \times 90 \mathrm{mmHg}$ no dia do exame ou em uso de medicações anti-hipertensivas ${ }^{17}$.

Foram considerados diabéticos os pacientes com diagnóstico de diabetes melito, registrado em prontuário ou em uso regular de hipoglicemiantes ${ }^{18}$.

Após a entrevista, realizou-se o exame físico, com a coleta de dados antropométricos. 
Em um leito da instituição de saúde, com o paciente na posição de decúbito dorsal por, no mínimo, cinco minutos, previamente à sessão de hemodiálise (no caso do grupo de hemodialisados), realizou-se a aferição da pressão arterial sistólica de cada artéria dos membros inferiores (artérias tibial posterior e pediosa) e da braquial de cada membro superior (Grupo Controle) ou do contralateral à fístula arteriovenosa (hemodialisados).

A aferição da pressão arterial sistólica foi realizada duas vezes em cada artéria, por examinadores distintos, previamente treinados, utilizando-se o Doppler vascular portátil (modelo DV-2001, fabricado por Medpej em São Paulo, no Brasil) e um esfigmomanômetro calibrado (modelo aneroide mecânico, fabricado por $\mathrm{BD}^{\circledR}$, na Alemanha).

O ITB foi obtido pela divisão simples da maior pressão arterial sistólica obtida em cada artéria do membro inferior por aquela obtida nos membros superiores (Grupo Controle) ou aquela que não apresentava fístula arteriovenosa (hemodialisados).

Assim, com o intuito de aumentar a sensibilidade do método, foram obtidos até dois valores de ITB para cada membro inferior do paciente. Os índices obtidos foram incluídos no banco de dados, visto que, com a consideração apenas do índice obtido com a artéria de maior pressão em determinada extremidade, a prevalência de artérias pouco compressíveis ficaria subestimada, pois há pacientes que possuem ambas as alterações no mesmo membro.

Foram considerados anormais ITB inferior a 0,9 (indicativo de DAOP) ${ }^{1-3,8,11,15,19-26}$ ou superior a $1,3^{1,3,8,19,25}$ (indicativo de artéria pouco compressível). Valores de ITB entre 0,7 e 0,9 foram considerados indicativos de DAOP leve; entre $0,4 \mathrm{e}$ 0,7, DAOP moderada e abaixo de 0,4, DAOP severa ${ }^{24}$.

Foram designadas incompressíveis as artérias que não se comprimiram, ou seja, não apresentaram desaparecimento do som (fase $\mathrm{V}$ de Korotkoff), mesmo insuflando o manguito em $300 \mathrm{mmHg}$.

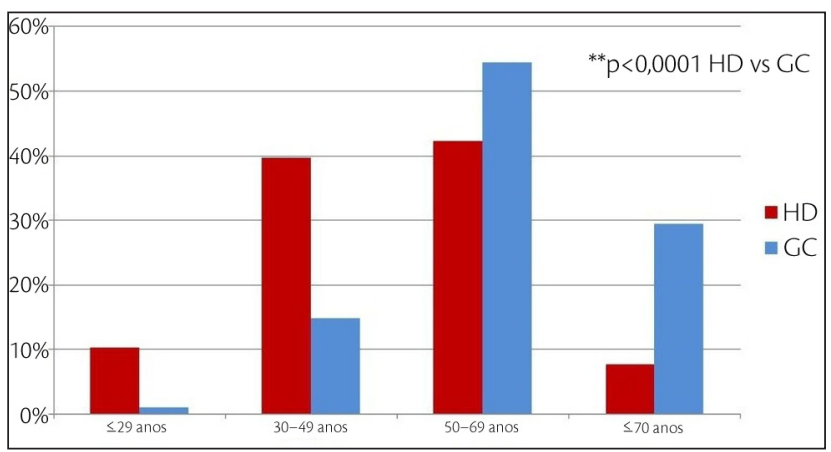

HD - pacientes em hemodiálise; GC - Grupo Controle.

Figura 1. Distribuição das faixas etárias entre os grupos.
Foram consideradas inaudíveis as artérias que não foram detectadas, por meio do Doppler vascular portátil, pelos dois examinadores.

Após colher as informações e examinar os pacientes, elaborou-se um banco de dados utilizando o programa Epi-Info Windows, versão 3.4.3, no qual foi realizada a análise estatística.

As variáveis qualitativas foram analisadas por meio dos testes do $\chi^{2}$ e Odds Ratio.

As variáveis numéricas foram analisadas por testes ANOVA, sendo observada a homogeneidade das variâncias pelo teste de Bartlett's. Assim, quando recomendado, utilizou-se o teste não paramétrico de Kruskal-Wallis.

Os resultados com probabilidade de erro inferior a $5 \%$ $(\mathrm{p}<0,05)$ foram considerados estatisticamente significantes.

\section{Resultados}

No grupo de hemodialisados, a média de idade foi de 49,07 $\pm 14,89$ (média - $\overline{\mathrm{X}} \pm$ desvio padrão - DP) e o tempo médio em hemodiálise foi de $36,90 \pm 35,65$ meses $(\overline{\mathrm{X}} \pm \mathrm{DP})$.

O sexo masculino e a raça branca predominaram entre o grupo de pacientes que participavam do programa de hemodiálise, com 49 e 34, respectivamente. Tal grupo era formado por $76(97,4 \%)$ hipertensos, $10(12,8 \%)$ tabagistas, $11(14,1 \%)$ ex-tabagistas e 24 (30,8\%) portadores de doença coronariana.

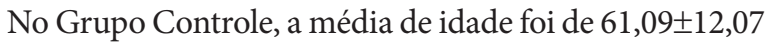
anos, com $52(59,1 \%)$ pacientes do sexo masculino; 42 $(47,7 \%)$, da raça branca; 57 (64,8\%), hipertensos; $14(15,9 \%)$, tabagistas; 34 (38,6\%), ex-tabagistas e $54(61,4 \%)$, portadores de doença coronariana, o que caracteriza um grupo de alto risco para doença vascular obstrutiva periférica.

As prevalências de diabetes melito e doença cerebrovascular foram semelhantes entre os grupos. Os dois grupos eram compostos por nove pacientes portadores de doença cerebrovascular e por quantidade semelhante de diabéticos, sendo 26 no grupo de pacientes em hemodiálise e 25 no Grupo Controle.

Tabela 1. Valores de pressão arterial sistólica e índice tornozelo-braço dos hemodialisados e Grupo Controle.

\begin{tabular}{lccc}
\hline Variáveis & HD & Grupo Controle & $\mathrm{p}$ \\
\hline PA MS - mmHg & $155,79 \pm 29,21$ & $144,13 \pm 25,64$ & $0,007^{\mathrm{a}}$ \\
PA MID - mmHg & $181,11 \pm 61,50$ & $155,26 \pm 38,91$ & $0,001^{\mathrm{k}}$ \\
PA MIE - mmHg & $177,9 \pm 53,82$ & $151,01 \pm 32,19$ & $0,000^{\mathrm{k}}$ \\
ITB MID & $1,17 \pm 0,37$ & $1,09 \pm 0,26$ & $0,066^{\mathrm{k}}$ \\
ITB MIE & $1,15 \pm 0,90$ & $1,06 \pm 0,50$ & $0,014^{\mathrm{k}}$ \\
\hline
\end{tabular}

HD - pacientes em hemodiálise; PA - pressão arterial; ITB - índice tornozelo-braço; MS membro superior; MID - membro inferior direito; MIE - membro inferior esquerdo; ateste ANOVA; kteste de Kruskal-Wallis; resultados expressos em média土desvio padrão. 
As medidas das pressões arteriais sistólicas encontram-se superiores ao recomendado pela Sociedade Brasileira de Hipertensão ${ }^{17}$ (Tabela 1).

Houve significância estatística ao serem analisadas as faixas etárias demonstradas na Figura 1.

Observou-se que $28(35,9 \%)$ hemodialisados possuíam os sinais e/ou sintomas pesquisados, no entanto, apenas $12(42,9 \%)$ apresentavam ITB inferior a 0,9 e $11(39,3 \%)$ possuíam ITB superior a 1,3. Assim, foi verificado que 21 (75\%) pacientes sintomáticos apresentaram ITB alterado com risco relativo de $1,79(\mathrm{p}=0,005)$.

Notou-se ainda que os cinco pacientes que apresentavam necrose tissular ou amputação de dedos, pés ou um dos membros (6,4\% dos hemodialisados) apresentaram valores alterados de ITB $(\mathrm{p}<0,05)$ e nenhum paciente relatou história de revascularização periférica.

Além disso, verificou-se que 21 pacientes, ou seja, metade dos hemodialisados com valores anormais de ITB eram assintomáticos, sendo que em oito destes a alteração do ITB foi inferior a 0,9 .

Não houve diferença estatística na prevalência de ITB alterado entre os grupos analisados, entretanto notou-se que artérias pouco compressíveis foram mais prevalentes nos hemodialisados e que a DAOP acometeu mais os pacientes do Grupo Controle (Tabela 2).

Tabela 2. Prevalência dos valores normais e alterados do índice tornozelo-braço.

\begin{tabular}{lccc}
\hline Interpretação do ITB & $\begin{array}{c}\text { HD } \\
\mathrm{n}(\%)\end{array}$ & $\begin{array}{c}\text { Grupo Controle } \\
\mathrm{n}(\%)\end{array}$ & $\mathrm{p}$ \\
\hline ITB normal & $36(46,2)$ & $44(50,0)$ & $\mathrm{NS}$ \\
DAOP & $21(26,9)$ & $29(33,0)$ & $\mathrm{NS}$ \\
$\begin{array}{l}\text { Artérias pouco } \\
\text { compressíveis }\end{array}$ & $24(30,8)$ & $20(22,7)$ & $\mathrm{NS}$ \\
Ambas alterações & $3(3,8)$ & $5(5,7)$ & $\mathrm{NS}$ \\
\hline
\end{tabular}

HD - pacientes em hemodiálise; ITB - índice tornozelo-braço; DAOP - doença arterial obstrutiva periférica; NS - não significativo estatisticamente; ${ }^{\mathrm{q}} \chi^{2}$.

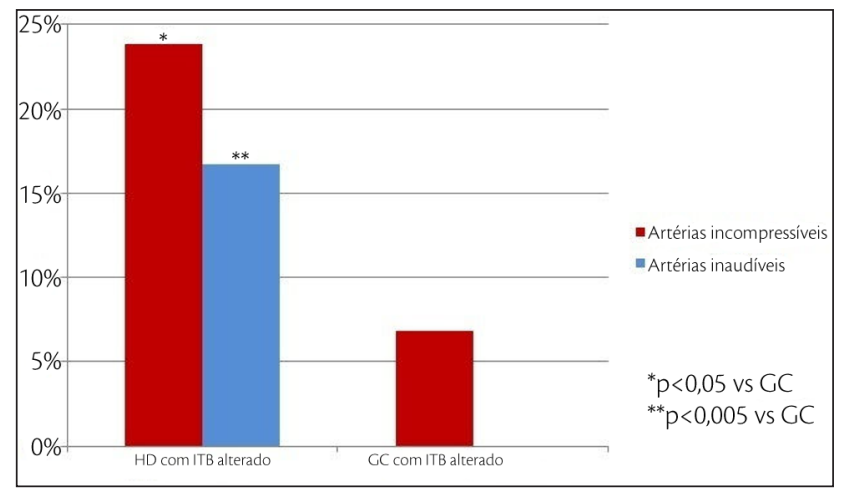

HD - pacientes em hemodiálise; GC - Grupo Controle; ITB - índice tornozelo-braço.

Figura 3. Prevalência de artérias incompressíveis e inaudíveis em pacientes em hemodiálise e no Grupo Controle.
Por intermédio das Figuras 2 e 3, observou-se que as alterações mais graves acometeram mais o grupo de hemodialisados do que o Grupo Controle. Desse modo, verificou-se que nenhum paciente do Grupo Controle apresentou ITB inferior a 0,4 , enquanto que $29 \%$ dos hemodialisados com DAOP (seis pacientes) apresentaram a doença em sua forma grave.

Mais da metade dos pacientes diagnosticados com DAOP no Grupo Controle possuía a doença em sua forma leve (25 pacientes); já no grupo de hemodialisados apenas 15 foram classificados desta maneira.

Além disso, dez $(23,8 \%)$ hemodialisados com valores anormais de ITB apresentaram artérias incompressíveis contra três $(6,8 \%)$, com ITB alterado do outro grupo e risco relativo de $3,49(\mathrm{p}<0,05)$.

Nenhum paciente do Grupo Controle apresentou artéria inaudível, enquanto que esta alteração foi observada em sete $(16,7 \%)$ hemodialisados, com valores anormais de ITB $(\mathrm{p}<0,005)$.

Como observado na Figura 4, o ITB alterado foi mais prevalente no sexo masculino do que no feminino nos dois grupos analisados, porém apenas houve significância estatística desta diferença no grupo de hemodialisados

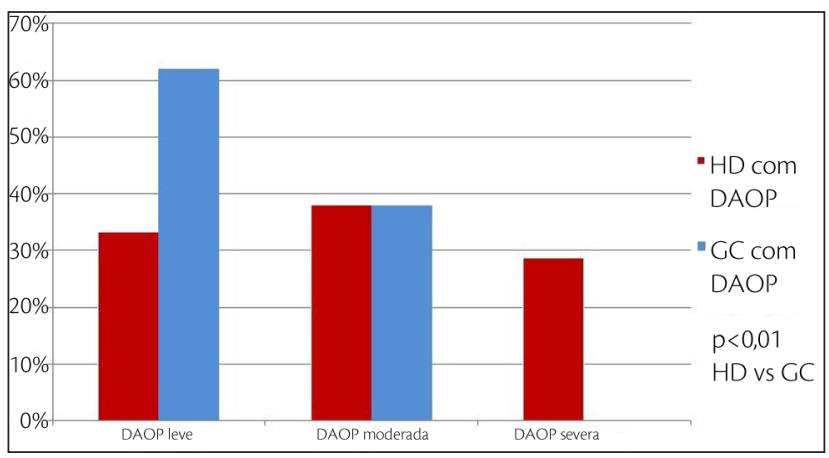

DAOP - doença arterial obstrutiva periférica; HD - pacientes em hemodiálise; CC - Grupo Controle. Figura 2. Prevalência dos graus de doença arterial obstrutiva periférica em pacientes em hemodiálise e o Grupo Controle.

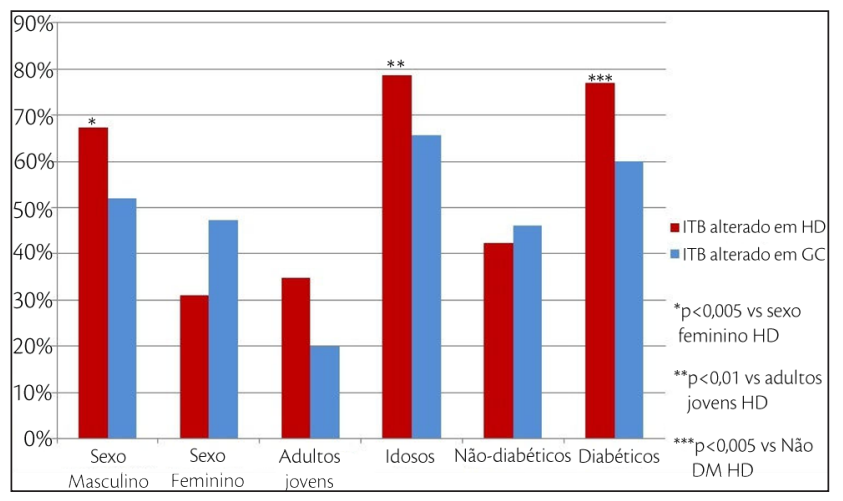

HD - pacientes em hemodiálise; GC - Grupo Controle; ITB - índice tornozelo-braço; DM - diabetes melito.

Figura 4. Distribuição de fatores de risco e variáveis correspondentes em pacientes em hemodiálise e no Grupo Controle com ITB alterado. 
$(\mathrm{p}<0,005)$. Neste grupo, verificou-se ITB alterado em 33 homens e 9 mulheres, com risco relativo de 1,77.

No grupo dos pacientes incluídos em programas de hemodiálise, também houve diferença estatisticamente significante entre os idosos e os adultos jovens com ITB alterado $(\mathrm{p}<0,01)$, o que não aconteceu no Grupo Controle, visto que tal grupo possuía apenas quatro indivíduos considerados adultos jovens. Dos 14 idosos que fizeram parte do primeiro grupo, $11(78,6 \%)$ apresentaram ITB anormal e, dos 23 adultos jovens que participavam deste grupo, oito $(34,8 \%)$ também apresentaram valores anormais deste índice.

Assim, verificou-se que os pacientes com 65 anos de idade ou mais apresentam maior probabilidade de apresentar ITB alterado do que aqueles com idades entre 20 e 39 anos e risco relativo de 2,26.

Outro importante fator de risco analisado no gráfico foi diabetes melito. Notou-se que a prevalência de alterações nos valores de ITB em diabéticos é superior a dos não diabéticos, sendo esta relação estatisticamente significante no grupo de hemodialisados $(\mathrm{p}<0,005)$. Neste grupo, 20 (76,9\%) diabéticos apresentaram ITB alterado, com risco relativo de 2,86.

Nos diabéticos de ambos os grupos, a prevalência de artérias pouco compressíveis também foi superior do que nos indivíduos não portadores de diabetes melito, com diferença estatisticamente significante apenas nos hemodialisados $(\mathrm{p}<0,01)$. Portanto, foram identificados sete diabéticos hemodialisados com artérias pouco compressíveis.

Observou-se que, nos pacientes que estavam incluídos no programa de hemodiálise por até cinco anos, a frequência de valores anormais de ITB aumentou gradativamente. A partir deste período, houve decréscimo desta frequência.

Assim, encontrou-se alteração do ITB em 51,6\% dos hemodialisados por até um ano; em $60 \%$ dos hemodialisados por um a três anos; em 64,3\% dos hemodialisados por três a cinco anos e em $50 \%$ dos hemodialisados por mais de cinco anos.

\section{Discussão}

A predominância do sexo masculino no grupo de hemodialisados foi semelhante a outros estudos com pacientes em regime de hemodiálise ${ }^{1,10,14}$, diferindo-se dos achados encontrados em estudos com pacientes pré-dialíticos ${ }^{19}$. Isto é explicado pelo fato de o homem evoluir mais para o estágio 5 da doença renal crônica do que a mulher, na qual o estrogênio funciona como fator protetor, favorecendo a angiogênese e dificultando a progressão da doença para a fase terminal ${ }^{27,28}$.

Tabagismo, sexo masculino, diabetes melito, idade avançada, doenças coronariana e cerebrovascular constituem variáveis que implicam risco para ITB alterado e elevada mortalidade ${ }^{1,6,9,14}$. Conforme indicado, o Grupo Controle apresentou maior prevalência destes fatores do que os hemodialisados, configurando um grupo de alto risco para DAOP.

A elevada prevalência de hipertensão arterial sistêmica e diabetes melito no grupo de hemodialisados, compatível com os estudos de Ono et al. ${ }^{14}$ e Longenecker et al. ${ }^{10}$, já era esperada pois estas são as principais etiologias de doença renal crônica ${ }^{29}$ e as principais doenças crônico-degenerativas do Brasil.

A elevada prevalência de ITB alterado nos dois grupos, sem diferença estatística entre eles, mesmo com o Grupo Controle apresentando mais fatores de risco, demonstra que a doença renal crônica em estágio terminal é um fator de risco importante para alteração do ITB, o que também foi observado por De Loach e Mohler ${ }^{1}$ e nas diretrizes K/DOQI ${ }^{13}$ e Transatlantic Inter-Society Consensus Document on Management of Peripheral Arterial Disease (TASC II) ${ }^{2}$.

Liew et al. ${ }^{24}$ afirmam que o portador de doença renal crônica e DAOP apresenta risco de morte significativamente maior do que aquele de uma das duas doenças isoladamente. Esse achado justifica o rastreio contínuo de DAOP e artérias pouco compressíveis nos pacientes em hemodiálise.

$\mathrm{O}$ fato de a doença renal crônica terminal representar fator de risco significativo para o desenvolvimento de doenças cardiovasculares pode ser explicado tanto por fatores de risco cardiovascular ditos tradicionais, quanto pelos não tradicionais que atuam na gênese da aterosclerose. Dentre os tradicionais, os mais prevalentes são diabetes, hipertensão, sedentarismo, hipertrigliceridemia, tabagismo e baixo $\mathrm{HDL}$, sendo o primeiro fator mais importante para a origem de doenças cardiovasculares e DAOP ${ }^{1,10,30-33}$.

Como foi possível notar, oito hemodialisados que foram diagnosticados com DAOP pelo ITB não apresentaram sinais ou sintomas desta doença. Esse fato mostra a superioridade do ITB em diagnosticar DAOP em relação ao método que é geralmente empregado na prática clínica (pesquisa de sintomas e palpação de pulsos das artérias dos membros inferiores). Além disso, isso nos faz refletir sobre a grande quantidade de pacientes com DAOP que passam despercebidos nos serviços de hemodiálise e que não utilizam rotineiramente o ITB.

A elevada prevalência de assintomáticos com alteração do ITB também foi descrita por O'Hare ${ }^{3}$, Carmo et al. ${ }^{19} \mathrm{e}$ Makdisse et al. ${ }^{11}$ e reforça o uso do ITB como o método de rastreio de DAOP.

Sete hemodialisados sintomáticos (25\%) apresentaram ITB normal. No entanto, os pacientes que apresentaram necrose tissular ou algum tipo de amputação tiveram ITB alterado.

A elevada prevalência de amputação encontrada em hemodialisados é atribuída à própria doença renal crônica 
e à elevada prevalência de diabetes melito ${ }^{1,2,34}$. A ausência de verificação rotineira do ITB dos pacientes nos serviços incluídos na pesquisa é outro fator que contribui para o excessivo número de amputados.

Como esperado, a idade avançada aumentou o risco de ITB alterado nos dois grupos, porém com maior prevalência nos hemodialisados. Os trabalhos de Ono et al. ${ }^{14}$, De Loach e Mohler ${ }^{1}$, Cheung et al. ${ }^{6}$ e Resnick et al. ${ }^{15}$ também mostram que idade avançada e prevalência de diabetes melito estão associadas com valores anormais de ITB.

Observou-se que, após o quinto ano de hemodiálise, houve decréscimo da prevalência das anormalidades pesquisadas. Isso está possivelmente relacionado à elevada mortalidade desta população, o que está em concordância com De Loach e Mohler ${ }^{1}$, Sesso ${ }^{35}$ e Silva et al. ${ }^{36}$, que apontam probabilidade de sobrevida em diabéticos hemodialisados por mais de cinco anos, de 25, 23 e 50\%, respectivamente ${ }^{1,35,36}$.

A prevalência de DAOP em hemodialisados foi de 26,9\%, o que está em concordância com O'Hare ${ }^{3}$, que afirma que este valor varia de 12 a $32 \%$.

No grupo de hemodialisados, $30,8 \%$ dos pacientes apresentaram artérias pouco compressíveis, valor um pouco acima do encontrado por um estudo finlandês, que relatou a prevalência estimada de artérias pouco compressíveis de $24 \%{ }^{3}$.

A maior prevalência de artérias pouco compressíveis verificada entre os hemodialisados é concordante com os dados apresentados por Ono et al. ${ }^{14}$ e se deve ao fato de pacientes com doença renal terminal possuírem grande distrofia óssea secundária ${ }^{7,37,38}$. Tais autores sugerem que a prevalência de DAOP pode ser subestimada, pois pacientes com artérias pouco compressíveis podem apresentar DAOP, apesar de seu ITB elevado ${ }^{25}$.

Foi observado que o grupo de hemodialisados apresentou lesões mais graves do que o Grupo Controle, inexistindo, neste último grupo, artérias inaudíveis ou DAOP grave. Esse é um dado muito importante, pois ITB menor que 0,5 em sintomáticos é um forte preditor de amputação².

\section{Conclusões}

A DAOP e as artérias pouco compressíveis foram muito prevalentes nos grupos estudados. No entanto, os pacientes hemodialisados apresentaram maior número de alterações mais graves, como artérias não compressíveis, inaudíveis ou amputação, mesmo quando comparados aos não dialíticos com fatores de risco mais presentes, tais como idade avançada, tabagismo e doença coronariana.
O ITB anormal em hemodialisados, seja como indicativo de DAOP ou de artérias pouco compressíveis, esteve elevado nas variáveis: sexo masculino, diabetes e idade avançada.

Em metade dos pacientes diagnosticados com DAOP pelo ITB, a doença apresentou-se assintomática, valorizando o emprego desse índice como método de rastreio das alterações vasculares descritas.

Logo, sugere-se a utilização do ITB na avaliação rotineira dos pacientes em hemodiálise.

\section{Referências}

1. De Loach SS, Mohler ER. Peripheral arterial disease: a guide for nephrologists. Clin J Am Soc Nephrol. 2007;2:839-46. PMid:17699501. http://dx.doi.org/10.2215/CJN.04101206

2. Norgren L, Hiatt WR, Dormandy JA, et al. Inter-Society Consensus for the Management of Peripheral Arterial Disease (TASC II). Eur J Vasc Endovasc Surg. 2007;33:S1-70. PMid:17223489.

3. O'Hare AM. Peripheral arterial disease in chronic kidney disease. [internet]. Up to date. 2009 [cited 2010 Feb 02]. Available from: http://www.utdol.com/patients/content/topic. do?topicKey= ROSOn9Yr_DYNx31.

4. Canziani MEF. Doenças Cardiovasculares na Doença Renal Crônica. J Bras Nefrol. 2004;26(3):20-1.

5. Martin LC, Franco RJS. Renal disease as a cardiovascular risk factor. Arq Bras Cardiol. 2005;85(6):1-5. http://dx.doi.org/10.1590/ S0066-782X2005001900011

6. Cheung AK, Sarnak MJ, Yan G, et al. Atherosclerotic cardiovascular disease risks in chronic hemodialysis patients. Kidney Int. 2000;58(1):353-62. PMid:10886582. http://dx.doi. org/10.1046/j.1523-1755.2000.00173.x

7. Canziani MEF, Moysés RMA. Calcificação Vascular na DRC. J Bras Nefrol. 2008;30(Suppl 2):23-6. http://dx.doi.org/10.1590/ S0101-28002011000200017

8. Vinuesa SG, Ortega M, Martinez P, Goicoechea M, Campdera FG, Luño J. Subclinical peripheral arterial disease in patients with chronic kidney disease: Prevalence and related risk factors. Kidney Int Suppl. 2005;(93):S44-7. PMid:15613068.

9. O'Hare AM, Hsu CY, Bacchetti P, Johansen KL. Peripheral Vascular Disease Risk Factors among Patients Undergoing Hemodialysis. J Am Soc Nephrol. 2002;13(2):497-503. PMid:11805180.

10. Longenecker JC, Coresh J, Powe NR, et al. Traditional cardiovascular disease risk factors in dialysis patients compared with the general population: the CHOICE study. J Am Soc Nephrol. 2002;13(7):1918-27.

11. Makdisse M, Pereira AC, Brasil DP, et al. Prevalência e fatores de risco associados à doença arterial periférica no projeto Corações do Brasil. Arq Bras Cardiol. 2008;91(6):402-14. http://dx.doi. org/10.1590/S0066-782X2008001800008

12. Hirsch AT, Haskal Z), Hertzer NR, et al. ACC/AHA 2005 Practice Guidelines for the management of patients with peripheral arterial disease (lower extremity, renal, mesenteric, and abdominal aortic): A collaborative report from the American Association for Vascular Surgery/Society for Vascular Surgery, Society for Cardiovascular Angiography and Interventions, Society for Vascular Medicine and Biology, Society of Interventional 
Radiology, and the ACC/AHA Task Force on Practice Guidelines. Circulation. 2005;113:1474-547. PMid:16990459.

13. National Kidney Foundation. K/DOQI Clinical practice guidelines for chronic kidney disease: Evaluation, classification and stratification. Am J Kidney Dis. 2002;39(2 Suppl 1):S1-266.

14. Ono K, Tsuchida A, Kawai H, et al. Ankle-brachial blood pressure index predicts all-cause and cardiovascular mortality in hemodialysis patients. J Am Soc Nephrol. 2003;14(6):1591-8. PMid:12761260. http://dx.doi.org/10.1097/01.ASN.0000065547.9825

15. Resnick HE, Lindsay RS, McDermott MM, et al. Relationship of high and low ankle brachial index to all-cause and cardiovascular disease mortality: the strong heart study. Circulation. 2004;109(6):733-9. http://dx.doi.org/10.1161/01. CIR.0000112642.63927.54

16. Chen S, Chang J, Hwang S, et al. Significant correlation between ankle-brachial index and vascular access failure in hemodialysis patients. Clin J Am Soc Nephrol. 2009;4(1):128-34. PMid:19141657.

17. Sociedade Brasileira de Cardiologia; Sociedade Brasileira de Hipertensão; Sociedade Brasileira de Nefrologia. V Diretrizes Brasileiras de Hipertensão Arterial. Hipertensão. 2006;9(4):121.

18. Sociedade Brasileira de Endocrinologia e Metabologia. Diabetes Mellitus: Classificação e Diagnóstico. Projeto Diretrizes da Associação Médica Brasileira e Conselho Federal de Medicina. 2004;1-8.

19. Carmo WB, Pinheiro HS, Bastos MG. Doença Arterial Obstrutiva de Membros Inferiores em Pacientes com Doença Renal Crônica Pré-Dialítica. J Bras Nefrol. 2007;29(3):127-34.

20. Sociedade Brasileira de Angiologia e Cirurgia Vascular. Diagnóstico da Doença Arterial Obstrutiva Periférica (DAOP). J Vasc Bras. 2005;4(3 Suppl 4):222-38.

21. Wittke El. Associação entre diferentes parâmetros de variabilidade da pressão sistólica fornecidos pela monitorização ambulatorial de pressão arterial (MAPA) e o índice tornozelo-braquial. [dissertação]. Porto Alegre (RS): Universidade Federal do Rio Grande do Sul; 2009.

22. Aragão JA, Reis FP, Borges RR, Aragão MECS, Nunes MAP, Feitosa VLC. Prevalência da doença arterial obstrutiva periférica em doentes com insuficiência renal crônica. J Vasc Bras. 2009;8(4):301-6. http://dx.doi.org/10.1590/S1677-54492009000400004

23. Santos RA, Boas LGCV, Osiro PM, Costa GM, Cordeiro JA, Martins JFV. A importância do índice tornozelo-braquial no diagnóstico da doença carotídea em pacientes hipertensos. Rev Soc Bras Clin Med. 2009;7(5):299-303.

24. Liew YP, Bartholomew JR, Demirjian S, Michaels J, Schreiber Junior M). Combined Effect of Chronic Kidney Disease and Peripheral Arterial Disease on All-Cause Mortality in a High-Risk Population. Clin J Am Soc Nephrol. 2008;3(4):1084-9. PMid:2440260. http:// dx.doi.org/10.2215/CJN.04411007

25. Miguel SJB. Calcificações vasculares em pacientes hemodialisados: Correlação entre os achados ultra-sonográficos, radiológicos e clínico-laboratoriais. [dissertação]. Niterói (RJ): Universidade Federal Fluminense; 2009 .

26. Gabriel AS, Serafim IPH, Freitas CEM et al. Doença arterial obstrutiva periférica e índice tornozelo-braço em pacientes submetidos à angiografia coronariana. Rev Bras Cir Cardiovasc. 2007;22(1):49-59. http://dx.doi.org/10.1590/S0102-76382007000100011

27. Kang D, Yu ES, Yoon K, Johnson RL. The impact of gender on progression of renal disease: potential role of estrogen-mediated vascular endothelial growth factor regulation and vascular protection. Am J Pathol. 2004;164(2):679-88. http://dx.doi.org/10.1093/ eurheartj/ehp007
28. Stenvinkel P, Wanner C, Metzger T, et al. Inflammation and outcome in end-stage renal failure: Does female gender constitute a survival advantage? Kidney Int. 2002;62(5):1791-8. PMid:12371981.

29. Takaoka HH. Perfil de pacientes diabéticos em diálise. J Bras Nefrol. 2009;31(2):100-4.

30. Dummer CD, Thomé FS, Veronese FV. Doença renal crônica, inflamação e aterosclerose: novos conceitos de um velho problema. Rev Assoc Med Bras. 2007;53(5):446-50. http://dx.doi.org/10.1590/ S0104-42302007000500022

31. Belkin $M$, Owens CD, Whittemore AD, Donaldson MC, Conte MS, Gravereaux E. Doença Oclusiva Arterial Periférica. In: Townsend CM, Beauchamp RD, Evers BM, Mattox KL, editores. Sabiston Tratado de Cirurgia: A Base Biológica da Prática Cirúrgica Moderna, $18^{\mathrm{a}}$ ed. Rio de Janeiro: Elsevier; 2010. p. 1823-58.

32. Keane WF, Brenner BM, Zeeuw D, et al. The risk of developing end-stage renal disease in patients with type 2 diabetes and nephropathy: The RENAAL Study. Kidney Int. 2003;63(4):1499-507. PMid:12631367. http://dx.doi. org/10.1046/j.1523-1755.2003.00885.x

33. Sarnak MJ, Levey AS, Schoolwerth AC, et al. Kidney Disease as a Risk Factor for Development of Cardiovascular Disease: A Statement From the American Heart Association Councils on Kidney in Cardiovascular Disease, High Blood Pressure Research, Clinical Cardiology, and Epidemiology and Prevention. Hypertension. 2003;42(5):1050-65. PMid:14581387

34. Romão Junior JE. Tratamento de substituição da insuficiência renal crônica. In: Lopes AC, editor. Tratado de clínica médica. São Paulo: Roca; 2006. p. 2810-3.

35. Sesso R. Epidemiologia da doença renal crônica no Brasil e sua prevenção [Internet]. Secretaria de Estado da Saúde; Coordenadoria de Controle de Doenças; Centro de Vigilância Epidemiologia. São Paulo, 2007 [cited 2010 Jan]. Available from: ftp://ftp.cve.saude. sp.gov.br/doc_tec/cronicas/irc_prevprof.pdf

36. Silva LAM, Mezzomo NF, Pansard HM, et al. Sobrevida em hemodiálise crônica: estudo de uma coorte de 1.009 pacientes em 25 anos. J Bras Nefrol. 2009;31(2):190-7.

37. Boaz M, Weinstein T, Matas Z, Green MS, Smetana S. Peripheral vascular disease and serum phosphorus in hemodialysis: A nested case-control study. Clin Nephrol. 2005;63(2):98-105. PMid:15730051.

38. McCullough PA, Agrawal V, Danielewicz E, Abela GS. Accelerated Atherosclerotic Calcification and Mönckeberg's Sclerosis: A Continuum of Advanced Vascular Pathology in Chronic Kidney Disease. Clin J Am Soc Nephrol. 2008;3(6):1585-98. PMid:18667741.

Correspondência
Mariane Torres Uchôa
Loteamento Jardim Petrópolis I, quadra CH, 207 - Jardim Petrópolis
CEP 57080-535 - Maceió (AL), Brasil
E-mail: marianeuchoa@gmail.com
Contribuições dos autores
Concepção e desenho do estudo: MTU, DNAO, MEP, DBD
Análise e interpretação dos dados: MTU, DNAO, MEP, JCC
Coleta de dados: MTU, DNAO, GSS
Redação do artigo: MTU, DNAO, MEP
Revisão crítica do texto: MTU, DNAO, MEP, MAMG
Análise estatística: MTU, DNAO, JCC
Responsabilidade geral pelo estudo: MEP
*Todos os autores leram e aprovaram a versão final submetida ao J Vasc Bras.

